\title{
EVALUATION FOR VARIOUS RESOURCE ALLOCATION METHODS FOR MULTIUSER-MIMO OFDMA SYSTEMS
}

\author{
Jingon Joung and Yong H. Lee \\ *School of Electrical Engineering and Computer Science \\ Korea Advanced Institute of Science and Technology (KAIST) \\ 373-1, Guseong-dong, Yuseong-gu, Daejeon, 305-701, Republic of Korea \\ TEL: +82-42-869-3437, FAX: +82-42-869-4030 \\ E-mail: yohlee@ee.kaist.ac.kr
}

\begin{abstract}
A simple algorithm to select user-pair and allocate frequency band is proposed for multiuser-MIMO OFDMA systems. For sake of comparison, various resource allocation algorithms, such as max-throughput, best-fit, first-fit, and random-fit algorithm, are examined with regard to the system throughput, computational complexity, and fairness among the users. Computer simulation results show that the first-fit algorithm can achieve a large reduction of computation for resource allocation as well as good fairness among users, with only a small reduction of throughput.
\end{abstract}

\section{INTRODUCTION}

Multiuser multiple-input multiple-output (MU-MIMO) techniques have been studied to increase the sum achievable rates in wireless communication. Among the existing MU-MIMO methods, linear processing techniques [1]-[4], such as zeroforcing (ZF) based joint-channel diagonalization (JCD) [1], [2] and minimum mean square error (MMSE)-based methods [3], [4], require simpler transceivers than those of nonliner processing. The ZF-based methods, which perfectly cancel inter-user-interferences, can obtain more throughput than MMSE-based methods when signal-to-noise ratio (SNR) is high. However, when SNR is low, MMSE-based methods that consider the noise level of the receiver can obtain more throughput than ZF-based methods [4]. This trade-off with respect to the SNR makes the operation of MU-MIMO systems complex. To avoid this trade-off problem a modified MMSEbased linear processing method has been proposed, which has merits of both the ZF- and MMSE-based methods [5]. To increase data rate for multimedia communications of next generation wireless communication devices, broadband communication techniques, such as orthogonal frequency division multiple access (OFDMA), have been integrated into the MU-MIMO method. As demonstrated by international standards, such as IEEE802.16 Air Interface for Fixed and Mobile Broadband Wireless Access Systems [6], IEEE802.20 [7], and Wireless World Initiative New Radio (WINNER) [8], it is inevitable that OFDMA and MIMO techniques will be combined.

In this paper, we examine the application of MU-MIMO OFDMA systems. For MU-MIMO, a simple ZF-based technique is employed and various resource allocation algorithms,

* This work was supported in part by the University Information Technology Research Center (ITRC) Program of the government of Korea and by Brain Korea 21 Project, The school of information technology, KAIST in 2007. such as max-throughput, best-fit, first-fit, and random-fit algorithms, are compared with respect to the system throughput, computational complexity, and fairness among the users. In this study, it is assumed that the supportable number of users in one frequency band is limited to two. Simulation results show that the first-fit algorithm can reduce computational complexity by $83.7 \%$ and can obtain $98.9 \%$ fairness among users with a sacrifice of an $7.5 \%$ rate reduction compared with the maxthroughput algorithm.

\section{Multiuser Mimo System Model}

The system configuration of a MU-MIMO downlink with $T$ users, $N_{T}$ transmit antennas, and $N_{R}$ receive antennas, is shown in Fig. 1. According to the MU-MIMO techniques, up to $N_{T}$ different users can be supported simultaneously by using the same frequency band. Then, the number of supportable users $K$ is limited by $N_{T} B$, where $B$ is the number of subbands for MU-MIMO. The MIMO channel for the $j$ th user and $b$ th subchannel band $(b \in\{1, \ldots, B\})$ is represented as a matrix $\mathbf{H}_{j}(b) \in \mathbb{C}^{N_{R} \times N_{T}}$, where the $(m, n)$ th entry represents the complex gain from the $n$th transmit antenna to the $m$ th receive antenna and is independent, identically distributed (i.i.d.) zero mean complex Gaussian random variables with a unit variance. It is assumed that the MIMO channel is static in the same frequency band, such as an adaptive modulation and coding (AMC) zone in IEEE802.16e [6] systems, and all $\left\{\mathbf{H}_{j}(b)\right\}, \forall j$ and $\forall b$, are known at the transmitter, while the $j$ th receiver only knows the $j$ th MIMO channel $\mathbf{H}_{j}(b), \forall b$. Also, it is assumed that $\mathrm{E}\left[\mathbf{x}(k) \mathbf{x}(k)^{H}\right]=\mathbf{I}_{L_{k}}$, where $\mathbf{x}(k)$, $\mathrm{E}[\cdot]$ and $\mathbf{I}_{L_{k}}$ denote the transmitted symbol vector, the expectation, and a $L_{k}$-dimensional identity matrix. The spatial multiplexing is performed by forming a vector signal $\mathbf{x}(k)$ with $L_{k}$ symbols, $\mathbf{x}(k) \in \mathbb{C}^{L_{k} \times 1}$, preprocessing each vector, and transmitting the elements of the resulting vector $\mathbf{T}_{k}(b) \mathbf{x}(k)$ through different antennas in the $b$ th subchannel band, where $\mathbf{T}_{k}(b) \in$ $\mathbb{C}^{N_{T} \times L_{k}}$ is the transmit processing matrix given by $\mathbf{T}_{k}(b)=$ $\mathbf{W}_{k}(b) \overline{\mathbf{V}}_{k}(b) \mathbf{E}_{k}(b)$. Here, $\mathbf{W}_{k}(b) \in \mathbb{C}^{N_{T} \times\left(N_{T}-\sum_{j=1, j \neq k} L_{j}\right)}$ is the matrix for the inter-user-interference (IUI) suppression, $\overline{\mathbf{V}}_{k}(b) \in \mathbb{C}^{\left(N_{T}-\sum_{j=1, j \neq k} L_{j}\right) \times L_{k}}$ is the preprocessor for diagonalizing the $k$ th user's MIMO channel $\mathbf{H}_{k}(b)$, and $\mathbf{E}_{k}(b) \in \mathbb{C}^{L_{k} \times L_{k}}$ is the diagonal matrix for power control. The preprocessed vectors for each user are combined to yield $\sum_{k=1}^{K} \mathbf{T}_{k}(b) \mathbf{x}_{k}(b)$ and then transmitted. At the $k$ th receiver using the $b$ th subchannel band, the received signal $\mathbf{H}_{k}(b) \sum_{j=1}^{K} \mathbf{T}_{j}(b) \mathbf{x}_{j}(b)+\mathbf{n}_{k}(b)$, where $\mathbf{n}_{k}(b) \in \mathbb{C}^{N_{R} \times 1}$ is a noise vector whose elements are i.i.d. with a zero mean and 

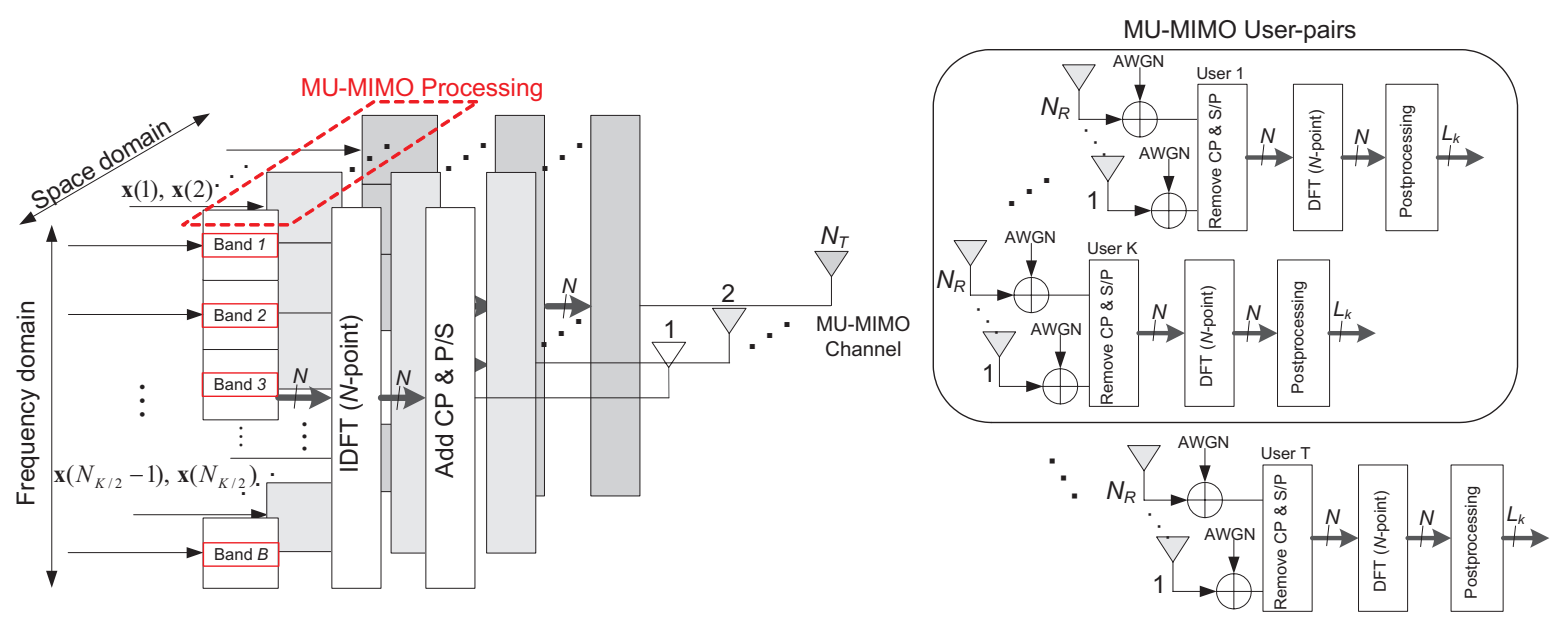

Figure 1: System configuration for multiuser MIMO-OFDMA downlink with $K$ users among total $T$ users.

variance $\sigma^{2}$, is postprocessed by $\mathbf{R}_{k}(b)^{H} \in \mathbb{C}^{L_{k} \times N_{R}}$ to yield

$$
\tilde{\mathbf{x}}_{k}(b)=\mathbf{R}_{k}(b)^{H} \mathbf{H}_{k}(b) \sum_{j=1}^{K} \mathbf{T}_{j}(b) \mathbf{x}_{j}(b)+\mathbf{R}_{k}(b)^{H} \mathbf{n}_{k}(b)
$$

where the superscript $H$ denotes the Hermitian transpose. The receive processing matrix $\mathbf{R}_{k}(b)^{H}$ is given by $\overline{\mathbf{U}}_{k}^{H}(b) \mathbf{U}_{k}^{H}(b)$ where $\mathbf{U}_{k}^{H}(b) \in \mathbb{C}^{L_{k} \times N_{R}}$ is the matrix combining $N_{R}$ received streams and $\overline{\mathbf{U}}_{k}^{H}(b) \in \mathbb{C}^{L_{k} \times L_{k}}$ is the postprocessor for diagonalizing the $k$ th user's MIMO channel $\mathbf{H}_{k}(b)$. When BD followed by singular value decomposition (SVD)-based singleuser MIMO processing is employed [1], [2], the IUI suppressing matrix becomes the nulling matrix as follows:

$$
\mathbf{W}_{k}(b)=\operatorname{null}\left(\mathbf{H}_{\bar{k}}^{H}(b) \mathbf{U}_{\bar{k}}^{H}(b)\right)
$$

where null $\{\cdot\}$ denotes the span of an orthogonal basis for the kernel or nullspace of the input matrix; $\mathbf{U}_{\bar{k}}(b)$ consists of $L_{\bar{k}}$ dominant left singular vectors of $\mathbf{H}_{\bar{k}}(b)$ where $\bar{k} \neq k$; and $\overline{\mathbf{V}}_{k}(b)$ and $\overline{\mathbf{U}}_{k}(b)$ are the right and left singular matrices of $\mathbf{U}_{k}^{H}(b) \mathbf{H}_{k}(b) \mathbf{W}_{k}(b)$, respectively. In this case, the IUI can be cancelled out completely based on a ZF criterion, and thus $\tilde{\mathbf{x}}_{k}(b)$ in (1) becomes

$$
\begin{aligned}
\tilde{\mathbf{x}}_{k}(b)= & \mathbf{R}_{k}^{H}(b) \mathbf{H}_{k}(b) \mathbf{T}_{k}(b) \mathbf{x}_{k}(b)+\mathbf{R}_{k}^{H}(b) \mathbf{n}_{k}(b) \\
= & \overline{\mathbf{U}}_{k}^{H}(b) \mathbf{U}_{k}^{H}(b) \mathbf{H}_{k}(b) \mathbf{W}_{k}(b) \overline{\mathbf{V}}_{k}(b) \mathbf{E}_{k}(b) \mathbf{x}_{k}(b) \\
& +\mathbf{R}_{k}^{H}(b) \mathbf{n}_{k}(b) \\
= & \overline{\mathbf{D}}_{k}(b) \mathbf{E}_{k}(b) \mathbf{x}_{k}(b)+\mathbf{R}_{k}^{H}(b) \mathbf{n}_{k}(b)
\end{aligned}
$$

where $\overline{\mathbf{D}}_{k}(b)$ is an $L_{k} \times L_{k}$ diagonal matrix consisting of the singular values of $\mathbf{U}_{k}^{H}(b) \mathbf{H}_{k}(b) \mathbf{W}_{k}(b)$, and the third equality comes from the fact that $\overline{\mathbf{U}}_{k}(b)$ and $\overline{\mathbf{V}}_{k}(b)$ are singular matrices of $\mathbf{U}_{k}^{H}(b) \mathbf{H}_{k}(b) \mathbf{W}_{k}(b)$.

\section{Multiuser PAir And BAnd Allocation}

The effective SNRs and the throughput ${ }^{1}$ for each subchannel are determined by the MU-MIMO user-pairs, and the achievable rates for the $k$-th user can be represented by $\omega\left(\mathbf{A}_{k}\right) \triangleq$

\footnotetext{
${ }^{1}$ For simplicity, we assumed that there is no power control, i.e., $\mathbf{E}_{k}(b)$ in (3) is a normalized identity matrix and up to two users are supportable simultaneously by using the same frequency band.
}

Table 1: Number of Real Operations for Computing $m_{i, j}(b)$

\begin{tabular}{c|c}
\hline$m_{i, j}(b)$ & \# of operations (addition plus multiplication) \\
\hline \hline$i \neq j$ & $6 L_{k} N_{R} N_{T}^{2}+24 N_{R} L_{k}^{2}-8 L_{k}^{3}$ \\
$i=j$ & $6\left(4 N_{R} N_{T}^{2}-4 N_{T}^{3} / 3\right)+1$ \\
\hline
\end{tabular}

$\sum_{i=1}^{L_{k}} \log _{2}\left(1+\lambda_{i}^{2} / \sigma^{2}\right)$, where $\lambda_{i}$ is the $i$ th largest singular value of an effective channel matrix $\mathbf{A}_{k}$. Then, we define the symmetric sum achievable rate matrix $\mathbf{M}(b)$; the $(i, j)$ th element of $\mathbf{M}(b), m_{i, j}(b)$, represents the sum achievable rates obtained by using the $b$ th subchannel band when combining the $i$ th and $j$ th users and it can be written as follows:

$$
m_{i, j}(b)= \begin{cases}\omega\left(\mathbf{H}_{i}(b) \mathbf{W}_{j}(b)\right)+\omega\left(\mathbf{H}_{j}(b) \mathbf{W}_{i}(b)\right), & i \neq j \\ \omega\left(\mathbf{H}_{i}(b) \mathbf{H}_{j}^{H}(b)\right), & i=j\end{cases}
$$

Here, the diagonal elements are achievable rates only when the $i$ th user is allocated at the frequency band $b$, i.e., a single user MIMO transmission. The computational complexity for $\mathbf{W}_{k}(b)$ in (2) is $\left(24 N_{T} N_{R}^{2}+48 N_{R}^{3}\right) B T$. To count the number of operations, we consider an efficient eigenvalue decomposition method in [9], which is based on the bidiagonalization and singular value decomposition. In addition, we treat that every complex operation as multiplication. Table 1 summarizes the number of operations associated with finding the sum achievable rates with given $\mathbf{H}_{k}(b)$ and $\mathbf{W}_{k}(b)$.

\section{A. Max-throughput Algorithm}

To obtain the optimal MU-MIMO user-pair selection, sum achievable rate matrices $\{\mathbf{M}(b)\}$ are required for the transmitter. Then, the optimal MU-MIMO user-pair set in terms of maximizing total-throughput is defined as

$$
\mathcal{S} \triangleq \underset{\{\mathcal{S}(b)\}}{\arg \operatorname{maximize}} \sum_{b} m_{i, j}(b)
$$

where MU-MIMO user-pair set $\mathcal{S}=\{\mathcal{S}(1), \cdots, \mathcal{S}(B)\}$ and $\mathcal{S}(b)$ represents the MU-MIMO user-pair $(i, j)$ for subchannel band $b$. Even though the optimal user-pair set $\mathcal{S}$ satisfying (5) can yield the maximum total-throughput, fairness among 
Table 2: Number of Computations for $m_{i, j}(b)$

\begin{tabular}{c|c|c}
\hline Algorithm & $m_{i, i}(b)$ & $m_{i, j}(b), i \neq j$ \\
\hline \hline Max-throughput & $T B$ & $B \sum_{i=1}^{T-1} i$ \\
(best-fit) & $\begin{array}{c}\left.\left\lceil\zeta \frac{K}{2}\right\rceil-1\right) B \\
\text { First-fit } \\
\text { Random-fit }\end{array}$ & $\sum_{i=1}^{\lceil T / 2\rceil-1}(T-(2 i-1))$ \\
\hline
\end{tabular}

users cannot be guaranteed at all. We call this method the maxthroughput algorithm, and this algorithm is summarized as follows:

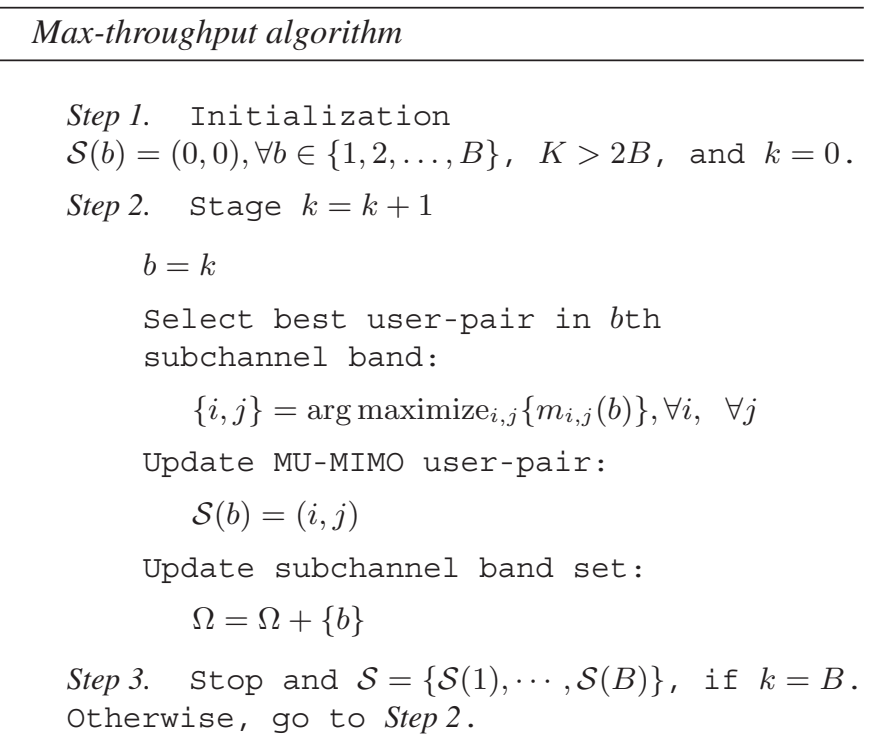

As we mentioned previously, the max-throughput algorithm cannot guarantee fairness among users, since the same user can be supported in the different scheduling stages termed $k$, i.e., some users multiply use the MU-MIMO bands. Here, if a certain scheduling algorithm, such as round robin or proportional fairness scheduling (see [10] and the references therein), is employed for selecting proper users, then fairness performance can be improved. However, the transmitter should know all $m_{i, j}(b)$ for all $i, j$, and $b$. This imposes a great burden of computational complexity for the transmitter. The total number of computations for $m_{i, j}(b)$ in (4) is summarized in Table 2, when $T$ is larger than two. Furthermore, even though $\{\mathbf{M}(b)\}$ are known at the transmitter, it is a formidable task to find the optimal $\mathcal{S}$ in terms of both the throughput and fairness, since so many combinations, $(T(T+1) / 2)^{B}$, should be compared to determine $\mathcal{S}$. Therefore, reducing the computational load for allocating resources is of practical importance.

\section{B. Best-fit Algorithm}

To avoid computational load due to the combinatorial search, the best-fit algorithm [11], which was proposed for a single carrier system, can be employed. The conventional best-fit algorithms utilize space-and-time resources for scheduling. On the other hand, the proposed best-fit algorithm utilizes spaceand-frequency resources by using $\{\mathbf{M}(b)\}$. The transmitter using the best-fit algorithm selects the user-pair which yields
Table 3: Simulation Parameters for MU-MIMO OFDMA System

\begin{tabular}{c|c}
\hline Parameter & Values \\
\hline \hline$\left\{N_{T}, N_{R}\right\}$ & $\{4,2\}$ \\
\# of subcarriers $N$ & 1024 \\
Carrier frequency & $2.3 \mathrm{GHz}$ \\
Sampling rates & $10 \mathrm{MHz}$ \\
Power profile (5 taps) & Pedestrian A in ITU \\
SNR & $25 \mathrm{~dB}$ \\
\# of OFDM symbols & 1000 \\
\# of MU-MIMO subchannel band $(B)$ & 6 bands \\
\hline
\end{tabular}

the highest throughput among all subchannel bands in every scheduling stage. Here, note that the users and subchannels selected in the $(k-1)$ th previous stage are discarded in the $k$ th present stage for fairness among users, which is similar to round robin scheduling. The best-fit algorithm is summarized as follows:

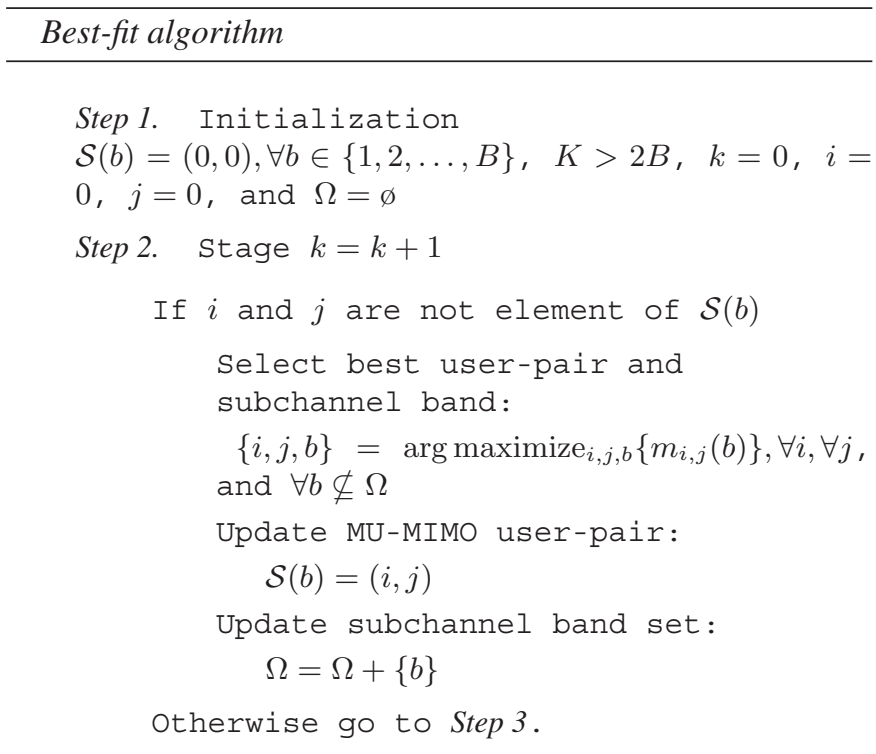

Step 3. Stop and $\mathcal{S}=\{\mathcal{S}(1), \cdots, \mathcal{S}(B)\}$, if $k=B$. Otherwise, go to Step 2 .

\section{First-fit Algorithm}

To further reduce computational complexity, the first-fit algorithm [12] can be employed in OFDMA systems. This algorithm can reduce computations by reducing the number of computations for $m_{i, j}(b)$ in (4) (see Table 2). The first-fit algorithm is described as follows: First, a transmitter decides the subchannel band for the 1st user. In this procedure, the transmitter compares $m_{1,1}(b)$ 's for all $b$, and then selects the subchannel $b^{\prime}$, which has the largest sum achievable rates, i.e., $b^{\prime}=\arg \max _{b}\left\{m_{1,1}(b)\right\}$. Secondly, in the allocated subchannel band $b^{\prime}$, all of $m_{a, j}\left(b^{\prime}\right)$ for all $j$ are compared, and then the transmitter decides the 1 st user's user-pair $\left(1, j^{\prime}\right)$, which has the largest sum achievable rates. Similarly, the transmitter can determine all user-pairs and their subchannels. Similarly to the best-fit algorithm, the previously selected users and allocated subchannels are discarded in the current resource allocation stage. The suboptimal first-fit user-pair selection algorithm 
Table 4: Computational Complexity for MU-MIMO User-pair Selection When $N_{T}=4, N_{R}=2$, and $L_{k}=2$

\begin{tabular}{c|c}
\hline Algorithm & \# of operations $(+, \times)$ when $T>2$ and $T$ is even \\
\hline \hline Max-throughput (best-fit) & $256 B T^{2}+771 B T$ \\
First-fit & $128 T^{2}+768 B T+259 B-771$ \\
Random-fit & $768 B$ \\
\hline
\end{tabular}

is summarized as follows:

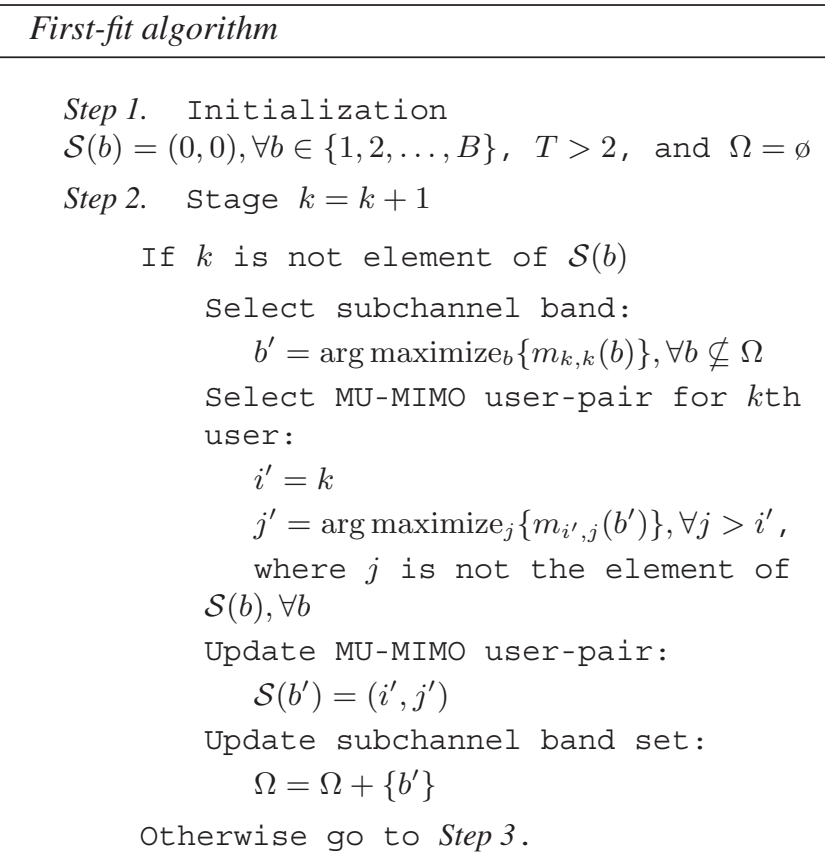

Step 3. Stop and $\mathcal{S}=\{\mathcal{S}(1), \cdots, \mathcal{S}(B)\}$, if $k=B$. Otherwise, go to Step 2 .

\section{Random-fit Algorithm}

To minimize computational complexity, both the user-pairs and subchannels can be allocated randomly. This algorithm is termed as the random-fit algorithm. Though this algorithm is the simpler than those mentioned previously (see Table 2), the resulting throughput would be seriously degraded. The random-fit algorithm is summarized as follows:

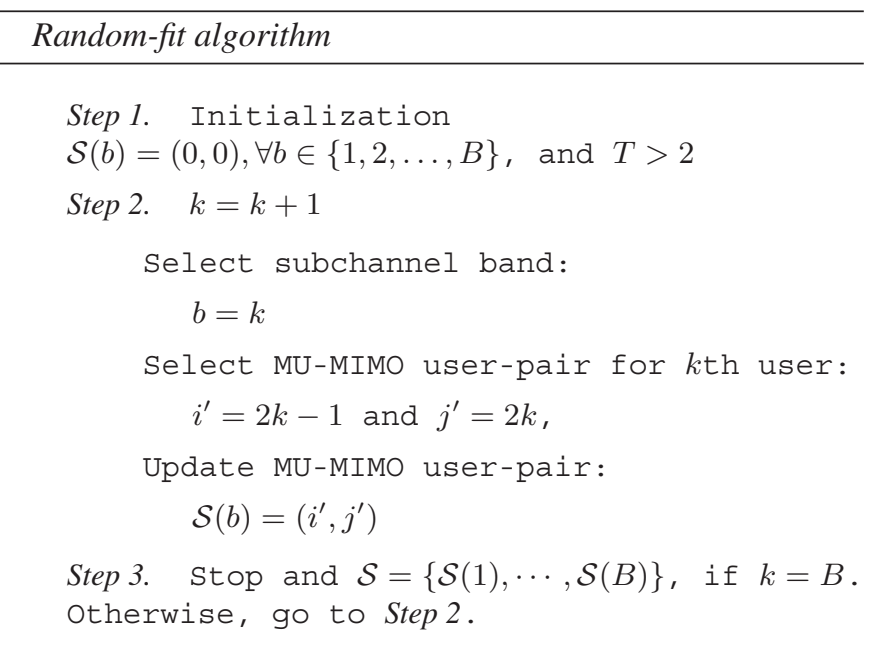

In this section, various resource allocation methods were examined in terms of the sum achievable rates, fairness among users, and computational complexity. The parameters for the simulation were summarized in Table 3. The MU-MIMO channel is obtained by generating independent Gaussian random variables with zero mean in the frequency domain, and the results shown below are the averages over 1000 independent trials. In the simulation, Jain's fairness index [13] was used to compare fairness among users. Jain's index $J$ is bounded between 0 and 1 . The higher index represents higher fairness among users. When there are $K$ users, Jain's index $J=\frac{\left(\sum_{k=1}^{K} r_{k}\right)^{2}}{K \sum_{k=1}^{K} r_{k}^{2}}$, where the normalized throughput $r_{k}=T_{k} / O_{k}, T_{k}$ is the measured throughput, and $O_{k}$ is the fair throughput, for the $k$ th user. Here, we assumed that all $O_{k}$ are identical for all $k$, i.e., traffic characteristics for each user are identical and required rates for each user are identical also.

By using Tables 1 and 2, the total numbers of operations to select MU-MIMO user-pairs are compared in Table 4 and illustrated in Fig. 2(a). Here, comparison complexities were not considered. Thus, the max-throughput and best-fit algorithms require the same computational complexities. The randomfit algorithm, which requires $\mathcal{O}(K)$ operations, provides great computational savings compared with the other algorithms. Both the best-fit and first-fit algorithms require $\mathcal{O}\left(T^{2}\right)$ operations in terms of $T$, yet the first-fit algorithm provides a savings in computation, since the order of $T^{2}$ operations for the first-fit algorithm is free from the $B$.

Figs. 2(b) and (c) show the average achievable rates and the Jain's fairness indices for four user-pair selection algorithms, respectively. It can be seen that the achievable rates, except for that of the random-fit algorithm, increase as the number of total users increases, i.e., only the random-fit algorithm cannot obtain multiuser diversity gain. Even though the max-throughput algorithm achieves the largest throughput among the various resource allocation algorithms, fairness among users is very poor. The fairness index of the max-throughput algorithm is 0.166 , which means that only $16.6 \%$ of users are happy. The second largest throughput is obtained by the best-fit algorithm. Unfortunately, the average fairness index of the best-fit algorithm is only about 0.709 . On the other hand, both the firstand random-fit algorithms provide about $98 \%$ fairness. Thus the first- and random-fit algorithms are worthwhile in terms of fairness.

In summary, when the total number of users is 32 , the random-fit algorithm obtained computational complexity reduction of about $99.7 \%$ against the max-throughput (bestfit) algorithm. However, the achievable rate decreased about 
Table 5: Performance Comparison Among Resource Allocation Methods

\begin{tabular}{c|cccc}
\hline Algorithms & MU diversity & Throughput & Complexity & Fairness \\
\hline \hline Max-throughput & $\bigcirc$ & Largest & Large & $16.6 \%$ \\
Best-fit & $\bigcirc$ & $-3.1 \%$ & Largest & $70.9 \%$ \\
First-fit & $\bigcirc$ & $-7.5 \%$ & Small & $98.9 \%$ \\
Random-fit & $\times$ & $-21.2 \%$ & Smallest & $98.4 \%$ \\
\hline
\end{tabular}

$21.2 \%$ from that of the max-throughput algorithm. In the firstfit algorithm case, an $83.7 \%$ reduction of computational complexity is accompanied by an $7.5 \%$ rate reduction. According to the computing power of the transmitter and required throughput, a user-pair selection algorithm can be determined (see Table 5). Here, it can be surmised that the best-fit algorithm is attractive in terms of fairness, computational complexity, and total-throughput.

\section{REFERENCES}

[1] Q. H. Spencer, A. L. Swindlehurst, and M. Haardt, "Zero-forcing methods for downlink spatial multiplexing in multiuser MIMO channels," IEEE Trans. Signal Processing, vol. 52, pp. 461-471, Feb. 2004.

[2] Z. Pan, K. K. Wrong, and T. S. Ng, "Generalized multiuser orthogonal space-division multiplexing," IEEE Trans. Wireless Commun., vol. 3, pp. 1969-1973, Nov. 2004.

[3] A. J. Tenenbaum and R. S. Adve, "Joint multiuser transmit-receive optimization using linear processing," in Proc IEEE Int. Conf. Commun. (ICC), Jun. 2004, vol. 1, pp. 588-592.

[4] J. Zhang, Y. Wu, S. Zhou, and J. Wang, "Joint linear transmitter and reciever design for the downlink of multiuser MIMO systems," IEEE Commun. Lett., vol. 9, pp. 991-993, Nov. 2005.

[5] J. Joung and Yong H. Lee, "Regularized channel diagonalization for multiuser MIMO downlink using a modified MMSE criterion," IEEE Trans. Signal Processing, vol. 55, pp. 1573-1579, Apr. 2007.

[6] IEEE Air Interface for Fixed and Mobile Broadband Wireless Access Systems, IEEE P802.16e/D12, 2005, Website http://ieeexplore.ieee.org.

[7] IEEE Mobile Broadband Wireless Access, IEEE 802.20, 2006, Website http://grouper.ieee.org/groups/802/20/2006.

[8] Wireless World Initiative New Radio (WINNER), 2006, Website https://www.ist-winner.org.

[9] G. H. Golub and C. F. Van Loan, Matrix Computation, 3rd ed. Baltimore, MD: The Johons Hopkins Univ. Press, 1996.

[10] J. Joung, Yong H. Lee, et al., "Capacity evaluation of various multiuser MIMO schemes in downlink cellular environments," in Proc. IEEE Personal, Indoor and Mobile Radio Communications (PIMRC), Helsinky, Finland, Sep. 2006.

[11] H. Yin and H. Liu, "Performance of space-division multiple-access (SDMA) with scheduling," IEEE Trans. Wireless Commun, vol. 1, pp. 611618, Oct. 2002.

[12] F. Shad, T. D. Todd, V. Kezys, and J. Litva, "Indoor SDMA capacity using a smart antenna base station," in Proc. IEEE International Conf. Universal Personal Commun. (ICUPC), Nov. 1997, pp. 868-872.

[13] R. Jain, W. Hawe, and D. Chiu, "A quantitative measure of fairness and discrimination for resource allocation in shared computer systems, Technical Report TR-301, DEC Research Labs, Sept. 1984.

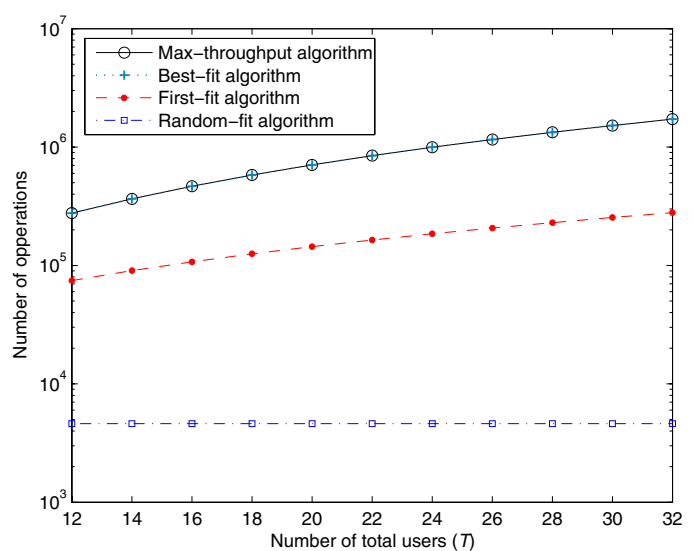

(a)

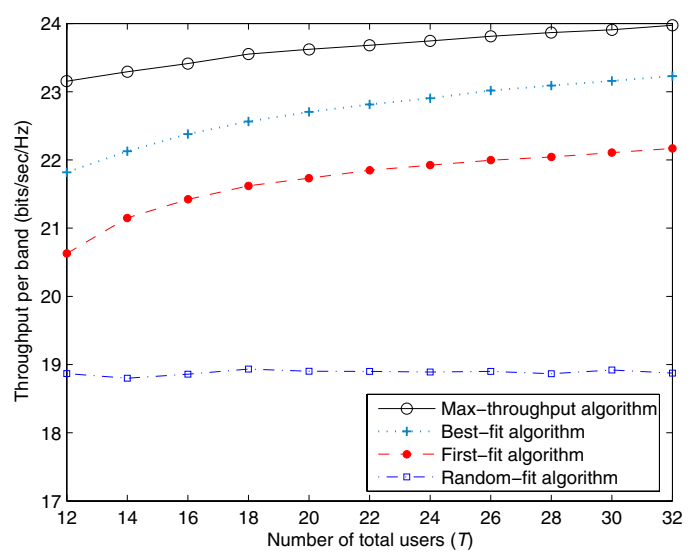

(b)

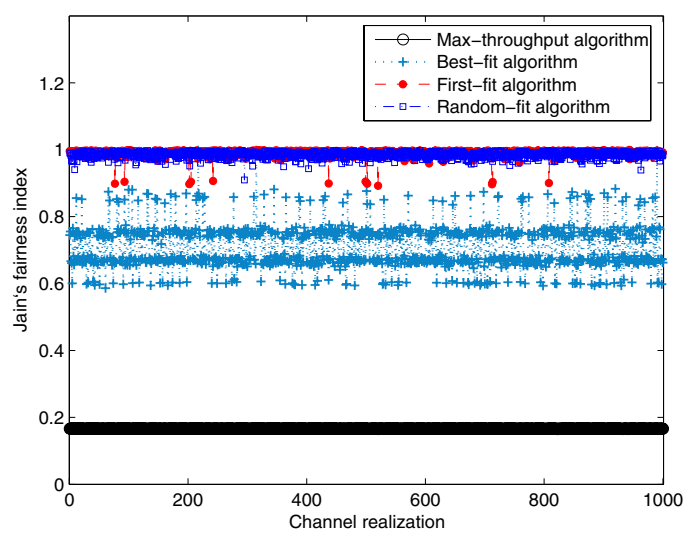

(c)

Figure 2: When $S N R=25 \mathrm{~dB}, B=6, N_{T}=4$, and $N_{R}=2$. (a) computational complexity comparison. (b) Sum achievable rates comparison. (c) Fairness comparison. 